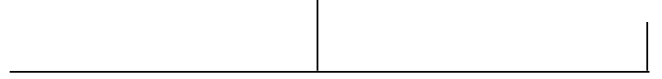

Rev. Latinoam. Psicopat. Fund., São Paulo, v. 11, n. 4, p. 573-587, dezembro 2008

\title{
Une physique du sens? Le problème de la formalization en psychanalyse
}

S. Askofaré, J.-L. Gaspard, P. Macary-Garipuy, M.-J. Sauret

L'enseignement de Lacan reposait au départ sur un axiome - "l'inconscient est structuré comme un langage" - et un programme: la formalisation de la psychanalyse. Cette entreprise le conduit dans un premier temps à une formalisation des lois $d u$ langage. En un second temps, Lacan définira la psychanalyse comme une praxis, un lien social fondé sur le langage, un discours. Même envisagée sous cet angle, sa formalisation reste nécessaire parce que solidaire de la "doctrine du mathème", dans une visée de transmission intégrale.

Mots clés: Discours, formalisation, langage, sens 


\section{Introduction}

Le projet d'une formalisation de la psychanalyse est une idée tout à fait neuve et récente en ce champ. Ni Freud ni ses élèves et collègues immédiats n’ont songé à une telle forme d'élaboration de l'expérience et du savoir psychanalytiques. La métapsychologie est restée la forme ultime de cette élaboration chez Freud comme chez Karl Abraham, Sandor Ferenczi, Otto Rank, Hans Sachs ou Ernest Jones. Quelques décennies plus tard, elle le restera chez Michaël Balint, Mélanie Klein, Donald.W. Winnicott ou Hans Hartmann pour en rester aux figures les plus marquantes de la discipline. À cette impasse faite sur la formalisation de la psychanalyse, on peut certes trouver des raisons biographiques, de formation, d'ambiance intellectuelle ou de goût épistémique personnel tant de Freud - qui fut pourtant un auditeur des cours de Brentano - que de ses épigones. La raison déterminante semble cependant la suivante: la scientificisation de la psychanalyse devait se réaliser par son inscription dans les sciences de la vie (Freud) ou dans les sciences humaines et historiques (Reik, Rank, Sachs). Or ni les unes ni les autres de ces sciences n'étaient animées par le projet de leur formalisation. L’épistémologie des disciplines comme la "philosophie spontanée des savants” (Althusser) les conduisaient plutôt à privilégier le rapport à l'expérimentation, aux sources et aux documents qu'à se mathématiser. La question de la formalisation en psychanalyse ne se posera donc que plus tardivement avec deux tentatives parallèles et opposées, celle de Lacan en France et celle de W. R. Bion (1962, 1963) en Angleterre. Parallèles, elles le furent, pour autant que jamais ces deux tentatives ne se rencontrèrent, ce qui eut été profitable pour la psychanalyse. Opposées, aussi, elles le furent dans la mesure où l'essentiel du travail de Bion a consisté en une littéralisation de l'empirique là où Lacan, privilégiant le fondement, s'est attaché à littéraliser la structure. De sorte qu'on peut affirmer que Lacan, contre l'avis de la quasi-totalité de ses collègues, considère que l'empirisme n'est pas nécessairement ce qui convient à la théorie psychanalytique. En effet, pour lui la théorie de la psychanalyse ne saurait consister à produire du savoir par induction à partir de cas particuliers. D'où son intérêt pour l'axio- 
matisation et sa méfiance à l'endroit de ce qu'il a pu appeler "la carte forcée de la clinique” (1966, 800). Dans le présent article, nous nous proposons de présenter et d'interroger certains aspects et moments de cette tentative lacanienne de formalisation de la psychanalyse ${ }^{1}$ afin d'en établir à la fois les raisons, la nécessité mais aussi les limites qui tiennent aux particularités du réel de la psychanalyse.

\section{Position du problème}

Partons de la question: qu'entendre par formalisation? Disons que dans son acception moderne - liée dès son apparition au développement de l'axiomatique et à l'étude des structures abstraites "multivalentes" (structures algébriques surtout, à l'origine) - la formalisation est la présentation des théories scientifiques dans le cadre d'un système formel, permettant de caractériser sans ambiguïté les expressions du langage et les règles de démonstration recevables (Ladrière, 1969, p. 109). Pour autant, on aurait tort de considérer que l'importance scientifique de la formalisation se réduise à une question de formulation ou de mise en formules. La formalisation des théories mathématiques fondamentales qui a conduit dans la seconde moitié du XXème siècle au développement de la logique mathématique comme discipline autonome - avec ses propres concepts et problèmes - a été suscitée à l'origine, on le sait, par les problèmes de "fondements" des mathématiques. ${ }^{2}$ Or, c'est une considération du même ordre que l'on retrouve à la base du projet lacanien de formalisation de la psychanalyse. Que "ce que Freud découvre, Lacan le fonde” a été longtemps, malheureusement, tenu pour un slogan. Il convient de revenir par conséquent au sérieux de ce dire, ne serait-ce que pour rappeler ceci: jamais Lacan ne s'est contenté d’une définition exclusivement clinique, donc empirique, de la psychanalyse. Freud non plus d'ailleurs si l'on prend comme référence la définition la plus exhaustive et la plus aboutie qu'il ait pu produire de la discipline dont il est l'inventeur (1985, p. 51). Pour autant, la

1. Chez Lacan cette question de la formalisation est de départ si l'on prend comme repère le fait qu'il date son enseignement de son discours de Rome de 1953, "Fonction et champ de la parole et du langage en psychanalyse” (1966, p. 237-322) et la conférence qui l’a précédée: “Le symbolique, l’imaginaire et le réel” (2005, p. 11-63).

2. Sur cette question des fondements des mathématiques, le lecteur se reportera avec profit à l'ouvrage de G. Frege, Les fondements de l'arithmétique, au n. 10 des Cahiers pour l'Analyse intitulé “La formalisation” et à la précieuse anthologie de l'Institut d’Histoire et Philosophie des Sciences et des Techniques, Logique et fondements des mathématiques (1992).

Rev. Latinoam. Psicopat. Fund., São Paulo, v. 11, n. 4, p. 573-587, dezembro 2008 
question se pose de savoir ce qui pourrait relever d'une entreprise de formalisation. Est-ce l'expérience, la pratique, la clinique? Est-ce la méthode, la technique? Est-ce le savoir, la théorie de l'expérience? Ou bien, est-ce la métathéorie, la structure? Assurément, Lacan a dû se heurter au même problème que W. Bion (1979), celui que F. Robert énonce très clairement dans son Avant-propos à l'édition française des Eléments de psychanalyse:

Si les descriptions cliniques sont déjà trop élaborées, trop théoriques, pour être recevables en tant que pures descriptions, les théories psychanalytiques, au contraire, parce qu'elles dérivent des observations faites et des expériences émotionnelles vécues dans l'analyse, demeurent souvent trop particulières, trop descriptives, pour être recevables en tant que théories universellement applicables. (p. 3)

En réponse au danger de "babélisation" de la psychanalyse, l' "abstractionnisme" de Bion est donc une tentative non axiomatique de formalisation, une littéralisation de l'expérience allant des éléments â au calcul algébrique, avec comme visée cette langue parfaite destinée à décrire et à transmettre intégralement et sans ambiguïté le savoir en tant que dépouillé de toute particularité, de toute marque énonciative. Entreprise fascinante et passionnante certes, mais qui reconduit dans la psychanalyse le rejet (ou forclusion) du sujet qui caractérise l'opération fondatrice de la science moderne. Cependant, on peut d'ores et déjà noter que ce n'est pas cette question pourtant cruciale du sujet, futur principe de séparation d'avec le structuralisme, qui va constituer à l'origine le principe de l'orientation lacanienne. Au départ, Lacan entreprend un double essai de formalisation: formalisation de l'expérience et de la clinique freudienne ${ }^{3}-$ formalisation de la théorie et de la structure. C'est à la dernière qu'il consacrera le plus gros de ses efforts et ce jusqu’à ses derniers séminaires topologiques. C'est également à cette dernière que nous allons exclusivement nous attacher désormais.

3. Il est assez aisé de se faire une idée de cette tentative de formalisation de la clinique dans "Le mythe individuel du névrosé ou Poésie et vérité dans la névrose” (1979), "Subversion du sujet et dialectique du désir” (1966, p. 793-827), ainsi que dans les Séminaires Livre IV - La relation d'objet (1956-57) et Livre V-Les formations de l'inconscient (1957-58). Ce projet, lié au "premier classicisme" (J.-C. Milner, 1995) trouve son accomplissement dans le graphe (complet) du désir. 


\section{Structure du langage et physique du sens}

Le pas décisif de Lacan, pensons-nous, a consisté à rapporter l'inconscient freudien à ses conditions de langage. C'est cette opération, sténographiée par les aphorismes devenus canoniques, qui supporte toute l'entreprise de formalisation initiée par Lacan. Il n'est inutile de revenir à ce moment fondateur qu'est le "Discours de Rome” (1953). Lacan y esquisse un programme étayé sur une épistémologie ou une doctrine de la science inspirée par A. Koyré. ${ }^{4}$ Son application à la psychanalyse comporte quelques conséquences - rejet de l'empirisme, démédicalisation, refus de l'objectivation abstraite - et une orientation: "le problème des fondements qui doivent assurer à notre discipline sa place dans les sciences: problème de formalisation, à la vérité fort mal engagé” (1966a, p. 284). Cette articulation du problème des fondements et de la formalisation implique et impose un détour par la linguistique. Il est ainsi visible que c'est d'un seul et même mouvement que Lacan place le langage au fondement de l'inconscient - et donc de la psychanalyse -, institue la linguistique, "science-pilote", en modèle - et peutêtre en rivale -, et adopte le paradigme structuraliste. Cette opération, qui dès lors fait relever la psychanalyse du "galiléisme étendu" (Milner, 1995), peut se condenser en trois propositions: de ce que le langage est structure, ses propriétés sont formalisables, mathématisables; ${ }^{5}$ les propriétés de l'inconscient, pour autant qu'il est structuré comme un langage, sont également formalisables, mathématisables; les propriétés formelles de l'inconscient-structure constituent un sous-ensemble limité des propriétés du langage. La voie est désormais ouverte pour le travail concret de formalisation de la psychanalyse (Lavendhomme, 2001).

\section{Formalisation de l'inconscient-structure}

Dès le texte "L'instance de la lettre dans l'inconscient ou la raison depuis Freud” (1966, p. 493-528), il apparaît nettement que ladite formalisation, d’une

4. Cette épistémologie de Koyré se déploie notamment dans: La révolution astronomique (1960), Etudes d'histoire de la pensée scientifique (1966a); Etudes galiléennes (1966b); Etudes newtoniennes (1968); Du monde clos à l'univers infini (1973).

5. Par mathématisable, il faut entendre ici : littéralisable, au sens où il s’agit d'une mathématique qui n’est pas centrée sur la quantité et qui ne vise pas la mesure ou la déduction. Ce qui n’exclut, bien au contraire, le calcul. Ainsi s'explique que ce soit à l'algèbre et à la topologie que Lacan va recourir pour mener à bien cette formalisation. 
part, doit excéder la formalisation de la linguistique comme telle et, d'autre part, ne peut être que partielle, "pas-toute" pour utiliser le vocable adéquat dans le lexique de Lacan. Pourquoi? Parce que la formalisation de la psychanalyse est tout à la fois et indissolublement formalisation du langage, de l'Autre, de l'inconscientstructure. ${ }^{6} \mathrm{C}^{\prime}$ est à cette formalisation de l'inconscient-structure via le langage que nous allons nous arrêter à présent pour en rappeler les articulations fondamentales. La construction repose sur les acquis de la linguistique et de la phonologie structurales, soit pour dire vite: le Cours de linguistique générale de F. de Saussure (1972) et Six leçons sur le son et le sens de R. Jakobson (1976). Le point de départ, une fois supposée la structure langagière de l'inconscient $(1966,495)$ est d'isoler l'atome du langage. Fidèle dans un premier temps à Saussure, Lacan l'identifie comme étant le signe, en tant qu'unité indissociable du signifiant et du signifié. S'émancipant de Saussure dans un second temps, Lacan interprète et radicalise l'invention saussurienne dont il fait non plus une unité insécable mais un rapport - entre deux éléments liés mais distincts: le signifiant $\mathrm{S}$ et le signifié s - mais un rapport problématique en ce que la barre - qui sépare les deux entités dans la topique qu'il propose n'est ni réductible à une barre de fraction ni équivalente à un symbole de représentation. Elle marque l'irréductibilité où se constituent les rapports du signifiant $\mathrm{S}$ au signifié s, la résistance de la signification. On remarquera que c'est à l'élément matériel, le signifiant, qu'est assigné le rôle causal. C'est le concept mathématique de fonction qui va servir d'opérateur catégoriel pour autoriser et réaliser les calculs requis par cette physique du sens. Le reste s'en déduit. Ainsi, les lois du langage, métaphore et métonymie qui avaient été dégagées par R. Jakobson trouvent à se calculer respectivement comme fonction de substitution et fonction de connexion du signifiant dans une chaîne (1966, p. 518). Deux questions restent cependant en suspens: en quoi ces fonctions du signifiant introduisent-elles et formalisent-elles quelque chose d'une "physique du sens", autrement dit d'un déterminisme des effets de langage? En quoi ces formules symbolisent-elles quelque chose des phénomènes auxquels la psychanalyse a affaire? Lacan prend son départ dans un concept du signifiant conçu comme atome du langage. Et c'est à ce signifiant dans sa matérialité spécifique qu'il assigne la fonction de poser un terme sur une barre résistante à la

6. Mais aussi chez Lacan d'autres formalisations qu'on pourrait dire locales: celle de la structure du sujet, des rapports à l'Autre et à l'autre (schéma $L$ ); de l'objet et des rapports à l'objet (logique du fantasme); de l'Oedipe et de la castration (métaphore paternelle); du lien social et en particulier du lien social analytique (théorie des discours); du choix sexué (formules de la sexuation); des registres de l'être, du symptôme et de la nomination (écriture borroméenne). 
signification. Les deux écritures fonctionnelles déduites de cette cellule algébrique attestent que dans le langage - procès d'écriture ou de parole - l'effet de signifié produit est fonction du mode d'agencement du signifiant. Ces modes d'agencement sont réduits aux deux mécanismes fondamentaux de la connexion et de la substitution signifiantes. Le calcul est peut-être élémentaire mais il y a calcul. Le problème, au regard de la psychanalyse, est que du coup cette "physique du sens” comme toute physique galiléenne forclôt le sujet. D’où la question que devra prendre en charge Lacan: comment intégrer le sujet dans ce procès du signifiant? Le rapport de ces formules avec les phénomènes auxquelles l'expérience psychanalytique a affaire passe par ce qu'il y a de plus fondamental dans cette pratique: le sens, l'interprétation, l'écriture, le chiffrage, le déchiffrage. ${ }^{7}$ Cette première tentative de formalisation de la psychanalyse fut donc pour l'essentiel sinon exclusivement une formalisation des lois du langage ${ }^{8}$ qui sont, répétons-le, les mêmes que les processus primaires freudiens, les lois de l'inconscient "travailleur idéal" donc. Par ailleurs, cette formalisation qui fournit la clef de la production des effets de signifié (effets de sens et effets de signification) fait l'impasse sur l'équivoque, pourtant si essentielle à la pratique comme à la théorie de l'interprétation, équivoque dont le concept est irréductible à la surdétermination. Enfin, au terme (plus logique que chronologique) d'une analyse, il y a l'acte analytique qui ne procède ni de métaphore ni de métonymie. L'acte, même de langage - et d'ailleurs saurait-il en exister d'autre? - requiert autre chose qu'une logique du signifiant et une physique du sens. C'est d'une authentique pragmatique de la signification qu'il va s'agir avec la théorie des discours qui constituera un nouveau chapitre de la formalisation lacanienne de la psychanalyse.

\section{Sens et signification à l'épreuve du discours}

En 1970, ce sera encore de nouveau à partir de la question des fondements - cette fois non pas de l'inconscient mais du lien social - que Lacan entreprendra sa formalisation de la psychanalyse dite aussi "théorie des quatre discours". Cette construction - envisagée par Lacan à la suite des évènements de Mai 1968

7. Référence de Lacan aux articles de G. Frege: "Fonction et concept” (1891) et “Qu'est-ce qu'une fonction?” (1904), recueillis plus tard dans Ecrits logiques et philosophiques (1971).

8. Nous disons bien les lois du langage (métaphore et métonymie) et non pas les lois de la parole qui sont autres et d’origine plutôt hégélienne (dissymétrie, médiation, reconnaissance). 
dans un colloque avec Freud, Wittgenstein et Foucault - prend son départ dans un examen du langage en tant que fondement du lien social qui fait tenir ensemble les corps. S'en déduisent des modes de liens sociaux et des effets de paroles déterminés par les contraintes qui président à la formation des structures ainsi constituées. ${ }^{9}$ Au sens où le construit Lacan, le discours est un assemblage de quatre éléments résultant d'une réduction littéral ${ }^{10}$ des constituants fondamentaux de la structure telle que déployée et théorisée à partir de l'expérience psychanalytique. La subversion produite par Lacan va consister à faire fonctionner de façon homogène ces éléments hétérogènes, en l'occurrence comme des lettres. Chacun de ces éléments peut occuper n'importe quelle place d'un discours dans la mesure où, justement, il n'est pas dépendant de celle-ci. Contrairement aux termes $\left(S_{1}, S_{2}, S\right.$, a) qui sont des objets littéraux, ${ }^{11}$ les places (agent ou semblant, autre, vérité, production) relèvent d'une logique modale de l'écriture et seront ordonnées à partir du carré logique (ou carré d'Apulée) et des modalités qui y sont à l'œuvre: nécessaire, impossible, contingent, possible..$^{12} \mathrm{~A}$ la différence des lettres qui oeuvrent à la "ronde des discours", les places discursives sont donc dépendantes. En effet, elles ne peuvent pas se subsister par elles-mêmes et donc ne peuvent être matériellement détachées du discours autrement que par abstraction. Comme les fonctions algébriques, pour exister matériellement, elles ont impérativement besoin d'être saturées. La question décisive à poser et à examiner dès

9. “Gouverner, éduquer, psychanalyser” sont trois tâches impossibles, disait Freud. Lacan s’est attaché à fonder en raison l'impossibilité de ces trois opérations - auxquelles il en ajoute une quatrième de son cru: faire désirer. De ces “choses folles”, comme il dit, Lacan fera rien moins que les quatre modes fondamentaux du lien social.

10. Qu'il suffise d'évoquer ici l’importance des catégories de Deutung (signifiance, interprétation), de Bedeutung (signification, référence, dénotation) et de Sinn (sens) dans l'œuvre freudienne pour attester que même si la psychanalyse n'est pas une herméneutique, la question du sens (donc du langage et de son procès) y est centrale. Le sens, dirons-nous, est ce qui dans une cure doit être traversé pour que le réel de la psychanalyse puisse être abordé.

11. Lesdits éléments sont: $S_{1}$ et $S_{2}$, c'est-à-dire le signifiant-maître et le savoir résumant la structure différentielle et oppositive du langage comme tel; $\$$ symbolise le sujet du signifiant que $\mathrm{S}_{1}$ représente auprès de $\mathrm{S}_{2}$; $a$ est la lettre qui inscrit la jouissance discursive dans son triple statut de jouissance produite - plus-de-jouir -, d’objet perdu et de cause du désir.

12. A la différence du carré logique, le tétraèdre de Lacan conjoint - et ne disjoint pas - le nécessaire et l'impossible, le contingent et le possible. En outre, Lacan fait le choix de subsumer en lieu et place de la modalité logique du possible, ce rapport sous la catégorie de l'impuissance qui traduit approximativement la barrière de la jouissance entre vérité et production, barrière qui constitue le poinçon de la castration qui structure tout discours. 
lors est la suivante: pourquoi cette catégorie de discours et cet effort pour la formaliser? On pourrait les rapporter à trois visées: La première est d'exploration de la dimension intermédiaire entre le langage et la parole. Dès son séminaire de 1968-1969, Lacan y a insisté: la psychanalyse ne peut pas se satisfaire du binarisme saussurien langage/parole ou du faux ternaire langage/langue/parole dans la mesure où la langue, en tant qu'institution, n'est que la forme empirique et historique que le langage prend pour une communauté déterminée. D’où la catégorie de discours qui est "une structure nécessaire qui dépasse de beaucoup la parole, toujours plus ou moins occasionnelle" (1991, p. 11). La thèse va même plus loin: Lacan considère en effet que "sans paroles" le discours peut fort bien subsister. La deuxième visée, appendue à la première, est relative à ce que traitent ces discours. Disons que les discours, tels que les conçoit la psychanalyse, sont d'abord des modes de traitement de la jouissance par le langage. Non pas de la jouissance en tant que telle, de la jouissance comme substance, mais de la jouissance en tant qu'elle manque, d'être exclue, séparée, structurellement disjointe du corps en tant qu'Autre (le corps comme désert de jouissance). D'où la nécessité de son rappel. Celui-ci s'opère selon deux voies fondamentales: la voie psychotique - hors discours - et la voie phallique: soit ce que nous rapportons classiquement à l'Oedipe et à la castration. Les discours constituent la modalité phallique - c'est-à-dire fondée sur la castration, la renonciation à la jouissance du passage de la jouissance à l'inconscient. Ce passage de la jouissance à l'inconscient, les discours l'effectuent en localisant la jouissance à sa place discursive (place dite de l'autre, de l'Autre ou du travail) et l'agent à la place maîtresse (dite aussi du semblant) qui commande l'opération de chacun des discours assurant et assumant la renonciation à cette jouissance. La troisième visée consiste à cerner comment les modes fondamentaux de liens sociaux déterminent les énonciations effectives mais également les significations, à l'intérieur des discours, et le sens, de la traduction d'un discours dans un autre. Ce projet vient s'inscrire comme réponse à la question: une "physique du sens", une doctrine du sens qui rend raison de sa production comme pur effet d'un mode d'agencement du signifiant suffit-elle à rendre compte de ce qui s'observe dans l'expérience analytique? C'est cette question, entre autres, que Lacan a été amené à relever et qui le conduira à cette formalisation nouvelle qui se situe au-delà de la logique du signifiant, au-delà de la structure de la parole et de ce qu'elle emporte de communication et de sémantique. En effet, dès "La science et la vérité”, Lacan renonce implicitement à son projet - celui du "Discours de Rome" - de faire de la psychanalyse une science. Ce renoncement n'est pas aveu d'impuissance mais reconnaissance in fine d'une spécificité et d'une altérité qui rendent la psychanalyse comme pratique irréductible à la science (1976, p. 53). Ce procès de "séparation" 
commence par une prise de distance vis-à-vis du structuralisme ${ }^{13}$ et culmine dans la théorie des quatre discours, donc dans l'invention de la catégorie de discours de l'analyste. Ce que la catégorie de discours prend en charge, c'est qu'il n'y a pas, d'une part, le langage et sa structure formelle - sans sujet -, et de l'autre la structure de la parole fondée sur le lien entre deux consciences. Du coup, langage et parole ne sont plus envisagés exclusivement à partir de leur pouvoir de signifier. User du langage, mettre en fonction la parole peut servir à bien d'autres choses qu'à signifier (Armengaud, 1999). C'est en ce point que la psychanalyse rencontre, entre autres, les questions dont s'occupe la pragmatique (Strawson, Bar-Hillel, Austin, Ducrot, Searle etc.) en tant qu'étude du langage "comme phénomène à la fois discursif, communicatif et social” (Jacques, 1984). Contentonsnous, dans le présent travail, de simplement situer comment la formalisation des discours subvertit la physique du sens et reconsidère les rapports entre sens et signification. Commençons par rappeler que l'opposition sens/signification - absente en tant que telle chez Freud -, bien qu'elle ait été thématisée par Frege depuis Über Sinn und Bedeutung (1892) n'est prise en charge que relativement tard par Lacan $(1957 ; 1958)$. Cette distinction timide s'effondre elle-même assez rapidement avec la promotion du syntagme de "signification phallique" - soit l'effet de signifié induit par la "métaphore paternelle" - qui viendra condenser le sens - rapportable à l'articulation signifiante, en particulier métaphorique - et la signification qui est, en l’occurrence, dénotation du phallus (non pas simplement comme signifiant mais aussi et surtout comme objet, manque d'objet). Mais avec l'invention de l'objet a, ce compromis implose. Si le fantasme que Lacan écrit ainsi : $\$<>$ a, est “index d'une signification absolue" $(1966,816)$, il résulte qu'aucune théorie de la référence n'est possible, en psychanalyse, sans l'objet a. En ambitionnant d'articuler "logique du signifiant" et "logique du fantasme", la catégorie de discours hérite du coup de l'antinomie qui se produit de signification à sens. À s'en tenir à la formalisation minimale que propose Lacan, on voit la difficulté qu'il y a à situer dans ce nouveau dispositif l'opposition signification/ sens. La signification est-elle appendue à l'objet - le plus-de-jouir - du discours et le sens à l'articulation des signifiants littéralisés que sont $S_{1}$ et $S_{2}$ ? Justement non. Et d'une certaine manière, on peut dire qu'au moins en partie l'intérêt de cette formalisation tient dans le fait qu'elle permet d'aller au-delà des solutions

13. La divergence décisive fut relative à la question du sujet et en particulier du sujet supposable à la structure de langage. Pour une reconstitution de ce débat, on se reportera au texte de Lacan de 1960, “Subversion du sujet et dialectique du désir” et au quatrième volumes des Mythologiques de Claude Lévi-Strauss, L’Homme nu, 1971. 
triviales. Au plus simple, nous dirons que la nouvelle écriture conduit à situer la signification comme interne au discours. Du simple fait de la mise en fonction particulière de la relation $S_{1} / S_{2}$ - relation dans laquelle, tout au moins s'agissant des trois discours non analytiques, $S 1$ précède et toujours détermine $S_{2}$, chaque discours engendre et n'engendre que de la signification; cette signification discursive est signification des dits dans lesquels baignent les sujets qui habitent ce discours mais à laquelle ils n'entendent rien! Le constat empirique du fait que c'est dans le discours analytique que se recueille et que peut être mis au jour le sens de l'inconscient met Lacan sur la voie de la modalité discursive de la production du sens: "le sens ne se produit jamais que de la traduction d'un discours en un autre” (2001, 480). Mais la traduction dont il s'agit n'est pas, loin s'en faut, traduction d'un système signifiant dans un autre, mais opération littérale qui fait passer d'un "système de lettres" à un autre (Allouch, 1984), et à quoi Lacan restitue son nom freudien: interprétation. C'est que sa signification, tout dit ne la tient que du moment où il n'avait pas de sens pour autant qu'aucun sens ne lui est consubstantiel. Ce sens donc ne peut lui provenir que du dehors, que de ce dehors qu'est pour lui un autre discours, le discours qui constitue son envers et qui est par là même est susceptible de l'interpréter. Parce que l'interprétation n'est rien d'autre que cela: “du sens qui va contre la signification” (2001, 480).

\section{Conclusion}

La question de la formalisation a donc à voir, nous pensons l'avoir établi, avec le statut de la psychanalyse dans le champ scientifique et le rapport de la psychanalyse à la science. Les deux “moments” que nous avons isolé dans le corpus lacanien témoignent par leur écart et leur différence d'une évolution voire d'une subversion de cette problématique. La première formalisation, celle des lois du langage et des modes de production du sens, illustre ce qui fut le moment d'aliénation de la psychanalyse à l'idéal de la science ; la deuxième - celle du discours - met au jour l'élaboration à quoi est rapportable le procès d'émancipation - séparation - de la psychanalyse à l'endroit de la science et celui de sa conceptualisation comme discours autonome. Il convient sans doute de prendre au sérieux le paradoxe qu'exhibent les résultats si opposés de ces deux formalisations. Nous pouvons en extraire a minima deux enseignements. Le premier est négatif; il consiste en une désidéalisation de la formalisation. Il nous permet de vérifier, en effet, ce que Lacan soulignait: "Une fausse science, comme une vrai, peut être mise en formules” (1973, p. 15). Le second, lui, est positif. Il atteste, nous semble-t-il, que la formalisation de la psychanalyse tient moins à son carac- 
tère "scientifique” qu’à son caractère logique (Laville, 1995, p. 282). Du coup nous comprenons, en même temps que le projet lacanien de mathématisation de la psychanalyse, le fait que la "mathématique lacanienne", dans son choix hyperbourbakiste (Milner, 1995, p. 136), épure la mathématique de sa déductivité - et de tout ce qu'elle peut charrier d'intuition et de référence à la quantité et à la mesure - pour ne s'intéresser qu'au “pur maniement des lettres” (Lacan, 1975, p. 47). Cette littéralisation, qui rapproche la psychanalyse des disciplines formelles (logique, mathématique), est aussi ce qui la fait tomber sous le coup du même verdict de non-scientificité. Car, ne l'oublions pas, pour nombre de scientifiques, la mathématique elle-même n'est pas une science (Feynman, 2000, p. 59). C'est sans doute pourquoi Lacan persévérera dans la voie d'une formalisation - sinon intégrale au moins locale - de la psychanalyse (1972-73, p. 108). Mais au-delà de l'élégance, de la rigueur et de la cohérence de ces formalisations, il restera surtout leur extraordinaire fécondité clinique et épistémique. Celle du graphe du désir sans doute (en tant que retombée de la formalisation de la logique du signifiant), mais aussi et surtout celle des discours pour leur contribution à l'intelligence du lien social et le nœud borroméen pour la lecture nouvelle qu'il autorise de la structure, des types cliniques voire de la fonction du symptôme.

\section{Références}

Allouch, J. Lettre pour lettre. Transcrire, traduire, translittérer. Toulouse: Erès, 1984. Armengaud, F. La pragmatique. Paris: PUF, 1999.

Bion, W. R. Learning from experience. New York: Basic Books, 1962. . Elements of psycho-analysis. New York: Basic Books, 1963. . Eléments de psychanalyse. Paris: PUF, 1979.

Feynman, R. P. Leçons de physique. Paris: Odile Jacob, 2000.

Frege, G. Les fondements de l'arithmétique. Paris: Seuil, 1969. . Ecrits logiques et philosophiques. Paris: Seuil, 1971.

Freud, S. Résultats, idées, problèmes II. Paris: PUF, 1985.

Jakobson, R. Six leçons sur le son et le sens. Paris: Editions de Minuit, 1976.

Koyre, A. La révolution astronomique. Paris: Hermann, 1960. . Etudes d'histoire de la pensée scientifique. Paris: Gallimard, 1966a. . Etudes galiléennes. Paris: Hermann, 1966b. 


\section{ARTIGOS}

. Etudes newtoniennes. Paris: Gallimard, 1968.

. Du monde clos à l'univers infini. Paris: Gallimard, 1973.

LaCAN, J. (1953). Fonction et champ de la parole et du langage en psychanalyse. In: Ecrits. Paris: Seuil, 1966. p. 237-322.

p. 493-528.

. (1957). L’instance de la lettre dans l’inconscient. In: Ecrits. Paris: Seuil, 1966.

. (1958). La signification du phallus. In: Ecrits. Paris: Seuil, 1966. p. 685-695.

. (1960). Subversion du sujet et dialectique du désir. In: Ecrits. Paris: Seuil, 1966. p. $793-827$.

. (1963). Kant avec Sade. In: Ecrits. Paris: Seuil, 1966. p. 765-790.

. (1964). Le séminaire. Livre XI. Les concepts fondamentaux de la psychanalyse. Paris: Seuil, 1973.

. Ecrits. Paris: Seuil, 1966.

. (1972-73). Le séminaire. Livre XX. Encore. Paris: Seuil, 1975.

. Conférences et entretiens dans les universités nord-américaines. Scilicet, Paris, n. 6/7, p. 5-63, 1976.

. Le mythe individuel du névrosé. Ornicar?, Paris, n. 17/18, p. 290-307, 1979.

. (1969-70). Le séminaire. Livre XVII. L'envers de la psychanalyse. Paris: Seuil, 1991.

. (1956-57). Le séminaire. Livre IV. La relation d'objet. Paris: Seuil, 1994.

. (1957-58). Le séminaire. Livre V. Les formations de l'inconscient. Paris: Seuil, 1998.

. L’Etourdit. In: Autres écrits. Paris: Seuil, 2001.

. Des Noms-du-Père. Paris: Seuil, 2005.

LADRIERE, J. Le théorème de Löwenheim-Skolem. Cahiers pour l'Analyse, Paris, n. 10, Le Graphe, p. 108-130, 1969.

Lavendhomme, R. Lieux du sujet. Paris: Seuil, 2001.

Laville, P. Le fil logique. In: Lacan avec les philosophes. Paris: Albin Michel, 1995.

Levi-Strauss, C. L’homme nu. In: Mythologiques. Paris: Plon, 1971. v. IV.

MiLneR, J.-C. 1995. L'œuvre claire. Paris: Seuil, 1995.

Rivenc, F.; De Rouilhan, P. Logique et fondements des mathématiques. Paris: Payot, 1992.

SAussure, F. Cours de linguistique générale. Paris: Payot, 1972. 


\section{Resumos}

(O físico do sentido? O problema da formalização da psicanálise)

O ensino de Lacan repousava, no início, sobre um axioma - "o inconsciente está estruturado como uma linguagem” - e um programa: a formalização da psicanálise. Esta tentativa o conduziu, num primeiro momento, a uma formalização das leis da linguagem. Posteriormente, Lacan definirá a psicanálise como uma práxis, um laço social fundado sobre a linguagem, um discurso. Contudo, mesmo considerada sob este ângulo, sua formalização continua sendo necessária, uma vez que é solidária à "doutrina do matema”, na perspectiva de uma transmissão integral.

Palavras-chave: Discurso, formalização, linguagem, sentido

(El fisico del sentido? El problema de la formalización del psicoanálisis)

La enseñanza de Lacan se basaba, en un comienzo, en un axioma - "el inconsciente está estructurado como un lenguaje" - y un programa: la formalización del psicoanálisis. Esta empresa lo conduce primeramente a una formalización de las leyes del lenguaje. Posteriormente Lacan definirá el psicoanálisis como una praxis, un lazo social fundado en el lenguaje, un discurso. Mismo considerándola bajo este aspecto, su formalización es todavía necesaria, puesto que es solidaria de la "doctrina del matema”, en una intención de transmisión integral.

Palabras claves: Discurso, formalización, lenguaje, sentido

(A physics of meaning? The problem of formalization in psychoanalysis)

Lacan's teaching was based on an axiom - "the unconscious is structured like a language" - and on a program - the formalization of psychoanalysis. Initially, this approach led to the formalization of the structure os language. Later, Lacan gave a great deal of attention to another approach: psychoanalysis is a praxis, a social link founded on language. In other words, is it a discourse. Even from this angle, its formalization does not remain less necessary because it is in line with the "theory of the matheme," from the point of view of full transmission.

Key words: Discourse, formalization, language, meaning

Versão inicial recebida em abril de 2007

Versão aprovada para publicação em setembro de 2008 


\section{Sidi AsKofaré*}

Maître de Conférences Université Toulouse 2, HDR, psychanalyste, Laboratoire de Psychopathologie clinique et psychanalyse de l'Université de Provence (E.A 3278)

3, place Victor Hugo

13331 Marseille Cedex 3

e-mail: s.askofare@wanadoo.fr

\section{JEAn-Luc Gaspard*}

Maître de Conférences Université Rennes 2, psychanalyste, Laboratoire Psychopathologie et Clinique Psychanalytique de l’Université Rennes 2 Haute Bretagne (E.A 4050)

place du Recteur Le Moal

35043 Rennes cedex

e-mail: jlgaspard@wanadoo.fr

Pascale Macary-Garipuy

Maître de Conférences Université Rennes 2, HDR, psychanalyste, Laboratoire Psychopathologie et Clinique Psychanalytique de l'Université Rennes 2 Haute Bretagne (E.A 4050)

place du Recteur Le Moal

35043 Rennes cedex

Marie-Jean Sauret

Professeur Université Toulouse 2, psychanalyste, Laboratoire de Psychopathologie clinique et psychanalyse de l'Université de Provence (E.A 3278)

3, place Victor Hugo

13331 Marseille Cedex 3

* Auteurs correspondants 